\title{
Kaon decays and the flavour problem
}

\author{
Gino Isidori \\ INFN, Laboratori Nazionali di Frascati, Via E. Fermi 40, I-00044 Frascati, Italy
}

\begin{abstract}
After a brief introduction to the so-called flavour problem, we discuss the role of rare $K$ decays in probing the mechanism of quark-flavour mixing. Particular attention is devoted to the formulation of the Minimal Flavour Violation hypothesis, as a general and natural solution to the flavour problem, and to the fundamental role of $K \rightarrow \pi \nu \bar{\nu}$ decays in testing this scenario.
\end{abstract}

\section{Introduction: the flavour problem}

Despite the Standard Model (SM) provides a successful description of particle interactions, it is natural to consider it only as the low-energy limit of a more general theory, or as the renormalizable part of an effective field theory valid up to some still undetermined cut-off scale $\Lambda$. Since the SM is renormalizable, we have no direct indications about the value of $\Lambda$. However, theoretical arguments based on a natural solution of the hierarchy problem suggest that $\Lambda$ should not exceed a few $\mathrm{TeV}$.

One of the strategies to obtain additional clues about the value of $\Lambda$ is to constrain (or find evidences) of the effective non-renormalizable interactions, suppressed by inverse powers of $\Lambda$, which encode the presence of new degrees of freedom at high energies. These operators should naturally induce large effects in processes which are not mediated by tree-level SM amplitudes, such as $\Delta F=1$ and $\Delta F=2$ flavour-changing neutral current (FCNC) transitions. Up to now there is no evidence of these effects and this implies severe bounds on the effective scale of dimension-six FCNC operators. For instance the good agreement between SM expectations and experimental determinations of $K^{0}-\bar{K}^{0}$ mixing leads to bounds above $10^{2} \mathrm{TeV}$ for the effective scale of $\Delta S=2$ operators, i.e. well above the few TeV range suggested by the Higgs sector.

The apparent contradiction between these two determinations of $\Lambda$ is a manifestation of what in many specific frameworks (supersymmetry, techincolour, etc.) goes under the name of flavour problem: if we insist with the theoretical prejudice that new physics has to emerge in the $\mathrm{TeV}$ region, we have to conclude that the new theory possesses a highly non-generic flavour structure. Interestingly enough, this structure has not been clearly identified yet, mainly because the SM, i.e. the low-energy limit of the new theory, doesn't possess an exact flavour symmetry. Then we should learn this structure from data, using the experimental information on FCNCs to constrain its form.

Recently the flavour problem has been considerably exacerbated by the new precise data of $B$-factories, which show no sizable deviations from SM expectations also in $B_{d}-\bar{B}_{d}$ mixing and in a clean $\Delta B=1 \mathrm{FCNC}$ processes such as $B \rightarrow X_{s} \gamma$. One could therefore doubt about the need for new tests of the SM in the sector of (quark) flavour physics. However, there are 


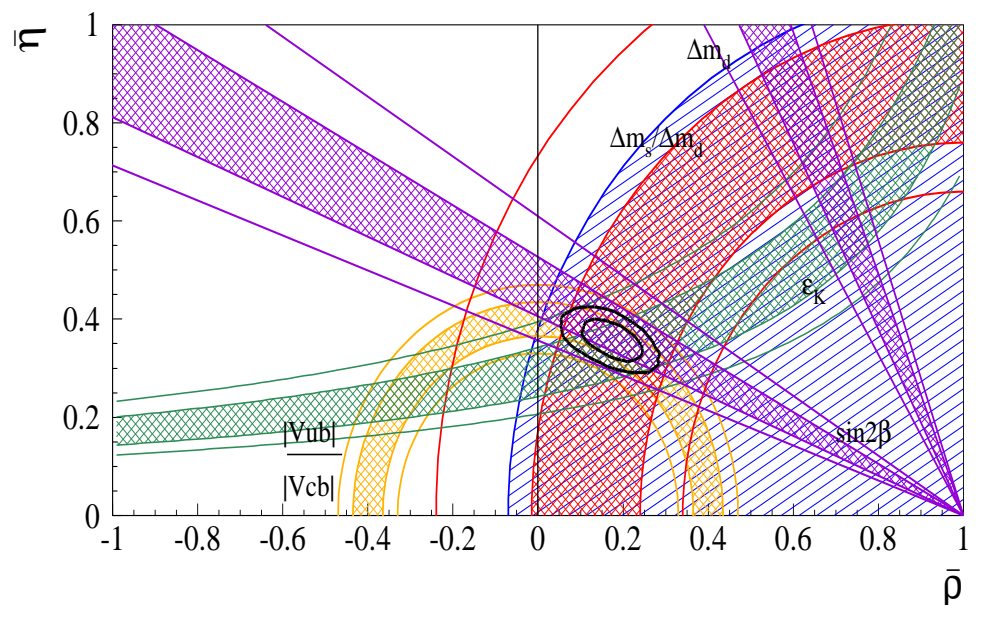

Figure 1: Allowed regions for the reduced Wolfenstein parameters $[2] \bar{\rho}$ and $\bar{\eta}(68 \%$ and $95 \%$ contours) compared with the uncertainty bands for $\left|V_{u b}\right| /\left|V_{c b}\right|, \epsilon_{K}, \Delta M_{B_{d}}$, the limit on $\Delta M_{B_{s}} / \Delta M_{B_{d}}$ and $a_{\mathrm{CP}}\left(B \rightarrow J / \Psi K_{S}\right)$ (from Ref. [3]).

at least two arguments why the present status cannot be considered conclusive and a deeper study of FCNCs is still very useful:

- The information used at present to test the CKM mechanism 1] and, in particular, to constrain the unitary triangle, is obtained only from charged currents (i.e. from tree-level amplitudes) and $\Delta F=2$ loop-induced processes (see Fig. 11). In principle, rare $K$ and $B$ decays mediated by $\Delta F=1$ FCNCs could also be used to extract indirect information on the unitary triangle, or to constrain new-physics effects. However, with the exception of the $B \rightarrow X_{s} \gamma$ rate, the quality of this information is very poor at present, either because of experimental difficulties or because of theoretical problems. Since new physics could affect in a very different way $\Delta F=2$ and $\Delta F=1$ loop-induced amplitudes [e.g. with $\mathcal{O}(100 \%)$ effects in the former and $\mathcal{O}(10 \%)$ in the latter], it is mandatory to improve the quality of the $\Delta F=1$ information.

- The most reasonable (but also most pessimistic) solution to the flavour problem is the so-called Minimal Flavour Violation (MFV) hypothesis. Within this framework, which will be discussed in detail in the next section, flavour- and CP-violating interactions are linked to the known structure of Yukawa couplings also beyond the SM. This implies that deviations from the SM in FCNC amplitudes rarely exceed the $\mathcal{O}(10 \%)$ level, or the level of irreducible theoretical errors in most of the presently available observables. Moreover, theoretically clean quantities such as $a_{\mathrm{CP}}\left(B \rightarrow J / \Psi K_{S}\right)$ and $\Delta M_{B_{d}} / \Delta M_{B_{s}}$, which measure only ratios of FCNC amplitudes, turn out to be insensitive to new-physics effects. Within this framework the need for additional clean and precise information on FCNC transitions is therefore even more important. As we shall discuss in the following, the measurements of $\Gamma(K \rightarrow \pi \nu \bar{\nu})$ would offer a unique opportunity in this respect (see Ref. 44 and references therein for a more extensive discussion). 


\section{The Minimal Flavour Violation hypothesis}

The pure gauge sector of the SM is invariant under a large symmetry group of flavour transformations: $G_{F} \equiv \mathrm{SU}(3)_{q}^{3} \otimes \mathrm{SU}(3)_{\ell}^{2} \otimes U(1)^{5}$, where $\mathrm{SU}(3)_{q}^{3}=\mathrm{SU}(3)_{Q_{L}} \otimes \mathrm{SU}(3)_{U_{R}} \otimes \mathrm{SU}(3)_{D_{R}}$, $\mathrm{SU}(3)_{\ell}^{2}=\mathrm{SU}(3)_{L_{L}} \otimes \mathrm{SU}(3)_{E_{R}}$ and three of the five $U(1)$ charges can be identified with baryon number, lepton number and hypercharge [5]. This large group and, particularly the SU(3) subgroups controlling flavour-changing transitions, is explicitly broken by the Yukawa interaction

$$
\mathcal{L}_{Y}=\bar{Q}_{L} Y_{D} D_{R} H+\bar{Q}_{L} Y_{U} U_{R} H_{c}+\bar{L}_{L} Y_{E} E_{R} H+\text { h.c. }
$$

Since $G_{F}$ is broken already within the SM, it would not be consistent to impose it as an exact symmetry of the additional degrees of freedom present in SM extensions: even if absent a the tree-level, the breaking of $G_{F}$ would reappear at the quantum level because of the Yukawa interaction. The most restrictive hypothesis we can make to protect the breaking of $G_{F}$ in a consistent way, is to assume that $Y_{D}, Y_{U}$ and $Y_{E}$ are the only source of $G_{F}$-breaking also beyond the SM.

To implement and interpret this hypothesis in a natural way, we can assume that $G_{F}$ is indeed a good symmetry, promoting the $Y$ to be dynamical fields with non-trivial transformation properties under $G_{F}$ :

$$
Y_{U} \sim(3, \overline{3}, 1)_{\mathrm{SU}(3)_{q}^{3}}, \quad Y_{D} \sim(3,1, \overline{3})_{\mathrm{SU}(3)_{q}^{3}}, \quad Y_{E} \sim(3, \overline{3})_{\mathrm{SU}(3)_{\ell}^{2}} .
$$

If the breaking of $G_{F}$ occurs at very high energy scales - well above the $\mathrm{TeV}$ region where the new degrees of freedom necessary to stabilize the Higgs sector appear - at low-energies we would only be sensitive to the background values of the $Y$, i.e. to the ordinary SM Yukawa couplings. Employing the effective-theory language, we then define that an effective theory satisfies the criterion of Minimal Flavour Violation if all higher-dimensional operators, constructed from SM and $Y$ fields, are invariant under $\mathrm{CP}$ and (formally) under the flavour group $G_{F}$ [5, 6].

According to this criterion one should in principle consider operators with arbitrary powers of the (adimensional) Yukawa fields. However, a strong simplification arises by the observation that all the eigenvalues of the Yukawa matrices are small, but for the top one, and that the off-diagonal elements of the CKM matrix $\left(V_{i j}\right)$ are very suppressed. It is then easy to realize that, similarly to the pure SM case, the leading coupling ruling all FCNC transitions with external down-type quarks is [6]:

$$
\left(\lambda_{\mathrm{FC}}\right)_{i j}= \begin{cases}\left(Y_{U} Y_{U}^{\dagger}\right)_{i j} \approx \lambda_{t}^{2} V_{3 i}^{*} V_{3 j} & i \neq j, \\ 0 & i=j .\end{cases}
$$

As a result, within this framework the bounds on the scale of dimension-six FCNC effective operators turn out to be much less severe than in the general case (see table 1).

The idea that the CKM matrix rules the strength of FCNC transitions also beyond the SM has become a very popular concept in the recent literature and has been implemented and discussed in several works (see e.g. Refs. [7]). However, it is worth to stress that the CKM matrix represent only one part of the problem: a key role in determining the structure of FCNCs is also played by quark masses, or by the Yukawa eigenvalues. In this respect the above MFV criterion provides the maximal protection of FCNCs (or the minimal violation of flavour symmetry), since the full structure of Yukawa matrices is preserved. We finally emphasize that, contrary to other approaches, the above MFV criterion is based on a renormalization-groupinvariant symmetry argument and is completely independent of specific new-physics framework. 


\begin{tabular}{lc|ccc}
$\begin{array}{c}\text { Minimally flavour violating } \\
\text { dimension six operator }\end{array}$ & $\begin{array}{c}\Lambda[\mathrm{TeV}] \\
-\end{array}$ \\
\hline$\frac{1}{2}\left(\bar{Q}_{L} \lambda_{\mathrm{FC}} \gamma_{\mu} Q_{L}\right)^{2}$ & $\epsilon_{K}, \quad \Delta m_{B_{d}}$ & 6.4 & 5.0 \\
$e H^{\dagger}\left(\bar{D}_{R} \lambda_{d} \lambda_{\mathrm{FC}} \sigma_{\mu \nu} Q_{L}\right) F_{\mu \nu}$ & $B \rightarrow X_{s} \gamma$ & 5.2 & 6.9 \\
$\left(\bar{Q}_{L} \lambda_{\mathrm{FC}} \gamma_{\mu} Q_{L}\right)\left(\bar{L}_{L} \gamma_{\mu} L_{L}\right)$ & $B \rightarrow(X) \ell \bar{\ell}, \quad K \rightarrow \pi \nu \bar{\nu},(\pi) \ell \bar{\ell}$ & 3.1 & 2.7 & $*$ \\
$\left(\bar{Q}_{L} \lambda_{\mathrm{FC}} \gamma_{\mu} Q_{L}\right)\left(H^{\dagger} i D_{\mu} H\right)$ & $B \rightarrow(X) \ell \bar{\ell}, \quad K \rightarrow \pi \nu \bar{\nu},(\pi) \ell \bar{\ell}$ & 1.6 & 1.6 & $*$
\end{tabular}

Table 1: $99 \%$ CL bounds on the scale of representative dimension-six operators in the MFV scenario [6]. The constraints are obtained on the single operator, with coefficient $\pm 1 / \Lambda^{2}(+$ or - denote constructive or destructive interference with the SM amplitude). The $*$ signals the cases where a significant increase in sensitivity can be achieved by future measurements of rare decays.

\section{$3 \quad K \rightarrow \pi \nu \bar{\nu}$ decays}

The $s \rightarrow d \nu \bar{\nu}$ transition is one of the rare examples of weak processes whose leading contribution starts at $\mathcal{O}\left(G_{F}^{2}\right)$. At the one-loop level it receives contributions only from $Z$-penguin and $W$ box diagrams, as shown in Fig. 2, or from pure quantum electroweak effects. Separating the contributions to the one-loop amplitude according to the intermediate up-type quark running inside the loop, we can write

$$
\mathcal{A}(s \rightarrow d \nu \bar{\nu})=\sum_{q=u, c, t} V_{q s}^{*} V_{q d} \mathcal{A}_{q} \sim \begin{cases}\mathcal{O}\left(\lambda^{5} m_{t}^{2}\right)+i \mathcal{O}\left(\lambda^{5} m_{t}^{2}\right) & (q=t) \\ \mathcal{O}\left(\lambda m_{c}^{2}\right)+i \mathcal{O}\left(\lambda^{5} m_{c}^{2}\right) & (q=c) \\ \mathcal{O}\left(\lambda \Lambda_{\mathrm{QCD}}^{2}\right) & (q=u)\end{cases}
$$

where $V_{i j}$ denote the elements of the CKM matrix. The hierarchy of these elements would favour up- and charm-quark contributions; however, the hard GIM mechanism of the perturbative calculation implies $\mathcal{A}_{q} \sim m_{q}^{2} / M_{W}^{2}$, leading to a completely different scenario. As shown on the r.h.s. of (4), where we have employed the standard CKM phase convention $\left(\Im V_{u s}=\Im V_{u d}=0\right)$ and expanded the $V_{i j}$ in powers of the Cabibbo angle $(\lambda=0.22)$, the top-quark contribution dominates both real and imaginary parts. This structure implies several interesting consequences for $\mathcal{A}(s \rightarrow d \nu \bar{\nu})$ : it is dominated by short-distance dynamics, therefore its QCD corrections are small and calculable in perturbation theory; it is very sensitive to $V_{t d}$, which is one of the less constrained CKM matrix elements; it is likely to have a large CP-violating phase; it is very suppressed within the SM and thus very sensitive to possible new sources of quark-flavour mixing.

Short-distance contributions to $\mathcal{A}(s \rightarrow d \nu \bar{\nu})$, both within the SM and within MFV models, can efficiently be described by means of a single effective dimension- 6 operator: $Q_{L}^{\nu}=$ $\bar{s}_{L} \gamma^{\mu} d_{L} \bar{\nu}_{L} \gamma_{\mu} \nu_{L}$. Within the SM both next-to-leading-order (NLO) QCD corrections [8, 9] and $\mathcal{O}\left(G_{F}^{3} m_{t}^{4}\right)$ electroweak corrections [10] to the Wilson coefficient of $Q_{L}^{\nu}$ have been calculated. The simple structure of $Q_{L}^{\nu}$ leads to two important properties of $K \rightarrow \pi \nu \bar{\nu}$ decays:

- The relation between partonic and hadronic amplitudes is exceptionally accurate, since hadronic matrix elements of the $\bar{s} \gamma^{\mu} d$ current between a kaon and a pion can be derived by isospin symmetry from the measured $K_{l 3}$ rates. 

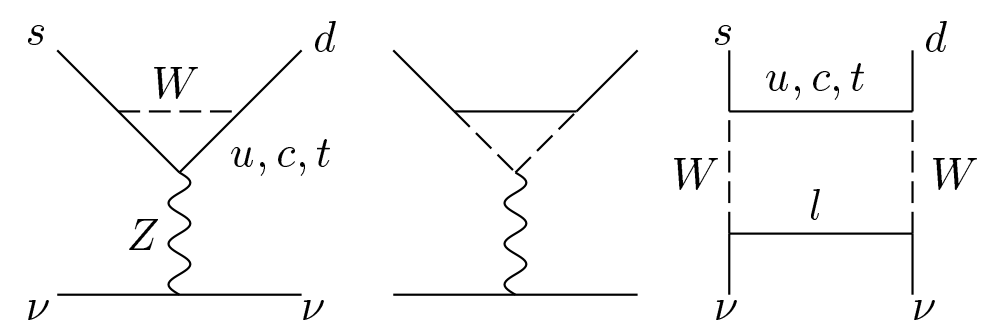

Figure 2: One-loop diagrams contributing to the $s \rightarrow d \nu \bar{\nu}$ transition.

- The lepton pair is produced in a state of definite CP and angular momentum, implying that the leading $\mathrm{SM}$ contribution to $K_{L} \rightarrow \pi^{0} \nu \bar{\nu}$ is CP-violating.

The dominant theoretical error in estimating the $K^{+} \rightarrow \pi^{+} \nu \bar{\nu}$ rate is due to the subleading, but non-negligible charm contribution. Perturbative NNLO corrections in the charm sector have been estimated to induce an error in the total rate of around $10 \%$, , which can be translated into a $5 \%$ error in the determination of $\left|V_{t d}\right|$ from $\mathcal{B}\left(K^{+} \rightarrow \pi^{+} \nu \bar{\nu}\right)$. Non-perturbative effects introduced by the integration over charmed degrees of freedom have been discussed in Ref. [11]: a precise estimate of these contributions is not possible, but they are expected to be within the error of NNLO terms. Genuine long-distance effects associated to light-quark loops have been shown to be much smaller [12].

The case of $K_{L} \rightarrow \pi^{0} \nu \bar{\nu}$ is even cleaner from the theoretical point of view [13]. Because of the CP structure, only the imaginary parts in (41) -where the charm contribution is absolutely negligible- contribute to $\mathcal{A}\left(K_{2} \rightarrow \pi^{0} \nu \bar{\nu}\right)$. Thus the dominant direct-CP-violating component of $\mathcal{A}\left(K_{L} \rightarrow \pi^{0} \nu \bar{\nu}\right)$ is completely saturated by the top contribution, where QCD corrections are suppressed and rapidly convergent. Intermediate and long-distance effects in this process are confined only to the indirect-CP-violating contribution [14] and to the CP-conserving one [15], which are both extremely small. Taking into account the isospin-breaking corrections to the hadronic matrix element [16], we can write an expression for the $K_{L} \rightarrow \pi^{0} \nu \bar{\nu}$ rate in terms of short-distance parameters, namely

$$
\mathcal{B}\left(K_{L} \rightarrow \pi^{0} \nu \bar{\nu}\right)_{\mathrm{SM}}=4.16 \times 10^{-10} \times\left[\frac{\bar{m}_{t}\left(m_{t}\right)}{167 \mathrm{GeV}}\right]^{2.30}\left[\frac{\Im\left(V_{t s}^{*} V_{t d}\right)}{\lambda^{5}}\right]^{2},
$$

which has a theoretical error below $3 \%$.

The high accuracy of the theoretical predictions of $\mathcal{B}\left(K^{+} \rightarrow \pi^{+} \nu \bar{\nu}\right)$ and $\mathcal{B}\left(K_{L} \rightarrow \pi^{0} \nu \bar{\nu}\right)$ in terms of modulus and phase of $\lambda_{t}=V_{t s}^{*} V_{t d}$ clearly offers the possibility of very interesting tests of flavour dynamics. Within the SM, a measurement of both channels would provide, two independent pieces of information on the unitary triangle, or a complete determination of $\bar{\rho}$ and $\bar{\eta}$ from $\Delta S=1$ transitions. As illustrated in Fig. 2, the high sensitivity of these two modes to short-distance dynamics makes them extremely efficient probes of MFV scenarios.

At present the SM predictions of the two $K \rightarrow \pi \nu \bar{\nu}$ rates are not extremely precise owing to the limited knowledge of $\lambda_{t}$. Taking into account all the indirect constraints in a global Gaussian fit, the allowed range reads [17, 18]

$$
\begin{aligned}
\mathcal{B}\left(K^{+} \rightarrow \pi^{+} \nu \bar{\nu}\right)_{\mathrm{SM}} & =(0.72 \pm 0.21) \times 10^{-10} \\
\mathcal{B}\left(K_{L} \rightarrow \pi^{0} \nu \bar{\nu}\right)_{\mathrm{SM}} & =(0.28 \pm 0.10) \times 10^{-10}
\end{aligned}
$$



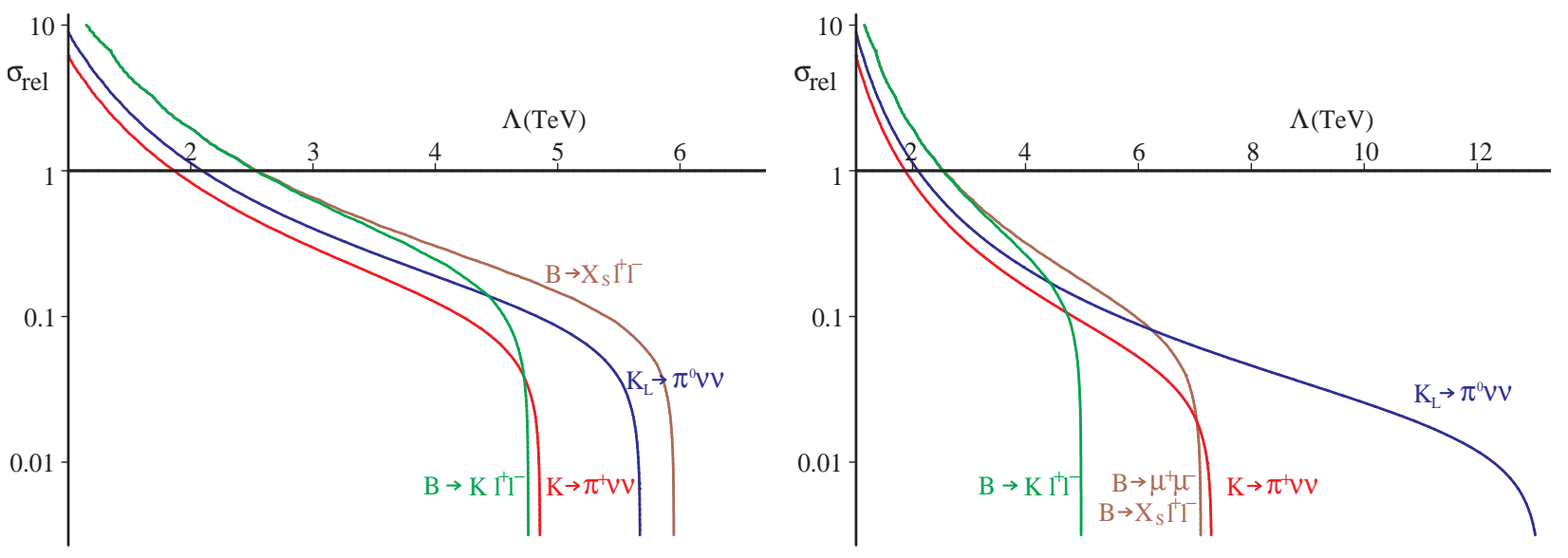

Figure 3: Comparison of the effectiveness of different rare modes in setting future bounds on the scale of the representative operator $\left(\bar{Q}_{L} \lambda_{\mathrm{FC}} \gamma_{\mu} Q_{L}\right)\left(\bar{L}_{L} \gamma_{\mu} L_{L}\right)$ within MFV models [6]. The vertical axis indicates the relative precision of an hypothetic measurement of the rate, with central value equal to the SM expectation. The curves in the two panels are obtained assuming an uncertainty of $10 \%$ (left) or 1\% (right) on the corresponding overall CKM factor.

The search for processes with missing energy and branching ratios below $10^{-10}$ is definitely a very difficult challenge, but has been proved not to be impossible: two $K^{+} \rightarrow \pi^{+} \nu \bar{\nu}$ candidate events have been observed by the BNL-E787 experiment [19]. The branching ratio inferred from this result,

$$
\mathcal{B}\left(K^{+} \rightarrow \pi^{+} \nu \bar{\nu}\right)=\left(1.57_{-0.82}^{+1.75}\right) \times 10^{-10},
$$

is consistent with SM expectations, although the error does not allow precision tests of the model yet. In a few years this result should be substantially improved by the BNL-E949 experiment, whose goal is to collect about 10 events (at the SM rate). In the longer term, a high-precision result on this mode will arise from the CKM experiment at Fermilab, which aims at a measurement of $\mathcal{B}\left(K^{+} \rightarrow \pi^{+} \nu \bar{\nu}\right)$ at the $10 \%$ level [20].

Unfortunately the progress concerning the neutral mode is much slower. No dedicated experiment has started yet (contrary to the $K^{+}$case) and the best direct limit is more than four orders of magnitude above the SM expectation [21. An indirect model-independent upper bound on $\Gamma\left(K_{L} \rightarrow \pi^{0} \nu \bar{\nu}\right)$ can be obtained by the isospin relation [22]

$$
\Gamma\left(K^{+} \rightarrow \pi^{+} \nu \bar{\nu}\right)=\Gamma\left(K_{L} \rightarrow \pi^{0} \nu \bar{\nu}\right)+\Gamma\left(K_{S} \rightarrow \pi^{0} \nu \bar{\nu}\right)
$$

which is valid for any $s \rightarrow d \nu \bar{\nu}$ local operator of dimension $\leq 8$ (up to small isospin-breaking corrections). Using the BNL-E787 result (8), this implies $\mathcal{B}\left(K_{L} \rightarrow \pi^{0} \nu \bar{\nu}\right)<1.7 \times 10^{-9}$ (90\% CL). Any experimental information below this figure can be translated into a non-trivial constraint on possible new-physics contributions to the $s \rightarrow d \nu \bar{\nu}$ amplitude. In a few years this goals should be reached by E931a at KEK: the first $K_{L} \rightarrow \pi^{0} \nu \bar{\nu}$ dedicated experiment. The only approved experiment that could reach the SM sensitivity on $K_{L} \rightarrow \pi^{0} \nu \bar{\nu}$ is KOPIO at BNL, whose goal is a SES of $10^{-13}$, or the observation of about 50 signal events (at the SM rate) with signal/background $\approx 2[20$. 


\section{Other Rare $K$ decays}

As far as theoretical cleanliness is concerned, $K \rightarrow \pi \nu \bar{\nu}$ have essentially no competitors. FCNC transitions where the neutrino pair is replaced by a charged-lepton pair have a very similar short-distance structure. However, in these processes the size of long-distance contributions is usually much larger because of electromagnetic interactions. Only in few cases (mainly in CPviolating observables) we can extract the interesting short-distance information with reasonable accuracy (see Ref. [20, 23] and references therein).

$\underline{K \rightarrow \pi \ell^{+} \ell^{-}}$The GIM mechanism of the $s \rightarrow d \gamma^{*}$ amplitude is only logarithmic [24]. As a result, the $K \rightarrow \pi \gamma^{*} \rightarrow \pi \ell^{+} \ell^{-}$amplitude is completely dominated by long-distance dynamics and provides a large contribution to the CP-allowed transitions $K^{+} \rightarrow \pi^{+} \ell^{+} \ell^{-}$and $K_{S} \rightarrow \pi^{0} \ell^{+} \ell^{-}$ 25. Rate and form factor of the charged mode have been measured with high accuracy by BNL-E865 [26]. However, this information is not sufficient to predict the rate of the neutral mode. The latter is usually parameterized as $\mathcal{B}\left(K_{S} \rightarrow \pi^{0} e^{+} e^{-}\right)=5 \times 10^{-9} \times\left|a_{S}\right|^{2}$ where $a_{S}$ is a low-energy free parameter expected to be $\mathcal{O}(1)$ [27]. The present experimental bound $\mathcal{B}\left(K_{S} \rightarrow \pi^{0} e^{+} e^{-}\right)<1.4 \times 10^{-7}$ [28] is still one order of magnitude above the most optimistic expectations, but a measurement or a very stringent bound on $\left|a_{S}\right|$ will soon arise from the $K_{S}$-dedicated run of NA48 and/or from the KLOE experiment at Frascati.

Apart from its intrinsic interest, the determination of $\mathcal{B}\left(K_{S} \rightarrow \pi^{0} e^{+} e^{-}\right)$has important consequences on the $K_{L} \rightarrow \pi^{0} e^{+} e^{-}$mode. Here the long-distance part of the single-photon exchange amplitude is forbidden by $\mathrm{CP}$ invariance and the sensitivity to short-distance dynamics in enhanced. The direct-CP-violating part of the $K_{L} \rightarrow \pi^{0} \ell^{+} \ell^{-}$amplitude is conceptually similar to the one of $K_{L} \rightarrow \pi^{0} \nu \bar{\nu}$ : it is calculable with high precision, being dominated by the top-quark contribution [29], and is highly sensitive to non-standard dynamics. This amplitude interfere with the indirect-CP-violating contribution induced by $K_{L}-K_{S}$ mixing, leading to [27]

$$
\mathcal{B}\left(K_{L} \rightarrow \pi^{0} e^{+} e^{-}\right)_{\mathrm{CPV}}=10^{-12} \times\left[15.3\left|a_{S}\right|^{2} \pm 6.8 \frac{\Im \lambda_{t}}{10^{-4}}\left|a_{S}\right|+2.8\left(\frac{\Im \lambda_{t}}{10^{-4}}\right)^{2}\right]
$$

where the \pm depends on the relative sign between short- and long-distance contributions, and cannot be determined in a model-independent way. Given the present uncertainty on $\mathcal{B}\left(K_{S} \rightarrow\right.$ $\pi^{0} e^{+} e^{-}$), at the moment we can only set a rough upper limit of $5.4 \times 10^{-10}$ on the sum of all the $\mathrm{CP}$-violating contributions to this mode, to be compared with the direct limit of $5.6 \times 10^{-10}$ obtained by $\mathrm{KTeV}$ at Fermilab [30].

An additional contribution to $K_{L} \rightarrow \pi^{0} \ell^{+} \ell^{-}$decays is generated by the CP-conserving longdistance processes $K_{L} \rightarrow \pi^{0} \gamma^{*} \gamma^{*} \rightarrow \pi^{0} \ell^{+} \ell^{-}$31. This amplitude does not interfere with the CP-violating one, and recent NA48 data on $K_{L} \rightarrow \pi^{0} \gamma \gamma$ (at small dilepton invariant mass) indicate that it is very suppressed, with an impact on $\mathcal{B}\left(K_{L} \rightarrow \pi^{0} e^{+} e^{-}\right)$below the $10^{-12}$ level 32 .

At the moment there exist no definite plans to improve the $\mathrm{KTeV}$ bound on $\mathcal{B}\left(K_{L} \rightarrow\right.$ $\left.\pi^{0} e^{+} e^{-}\right)$. The future information on $\mathcal{B}\left(K_{S} \rightarrow \pi^{0} e^{+} e^{-}\right)$will play a crucial role in this respect: if $a_{S}$ were in the range that maximizes the interference effect in (10), we believe it would be worthwhile to start a dedicated program to reach sensitivities of $10^{-12}$.

$K_{L} \rightarrow \ell^{+} \ell^{-}$Both $K_{L} \rightarrow \mu^{+} \mu^{-}$and $K_{L} \rightarrow e^{+} e^{-}$decays are dominated by the two-photon longdistance amplitude $K_{L} \rightarrow \gamma^{*} \gamma^{*} \rightarrow \ell^{+} \ell^{-}$. The absorptive part of the latter is determined to 

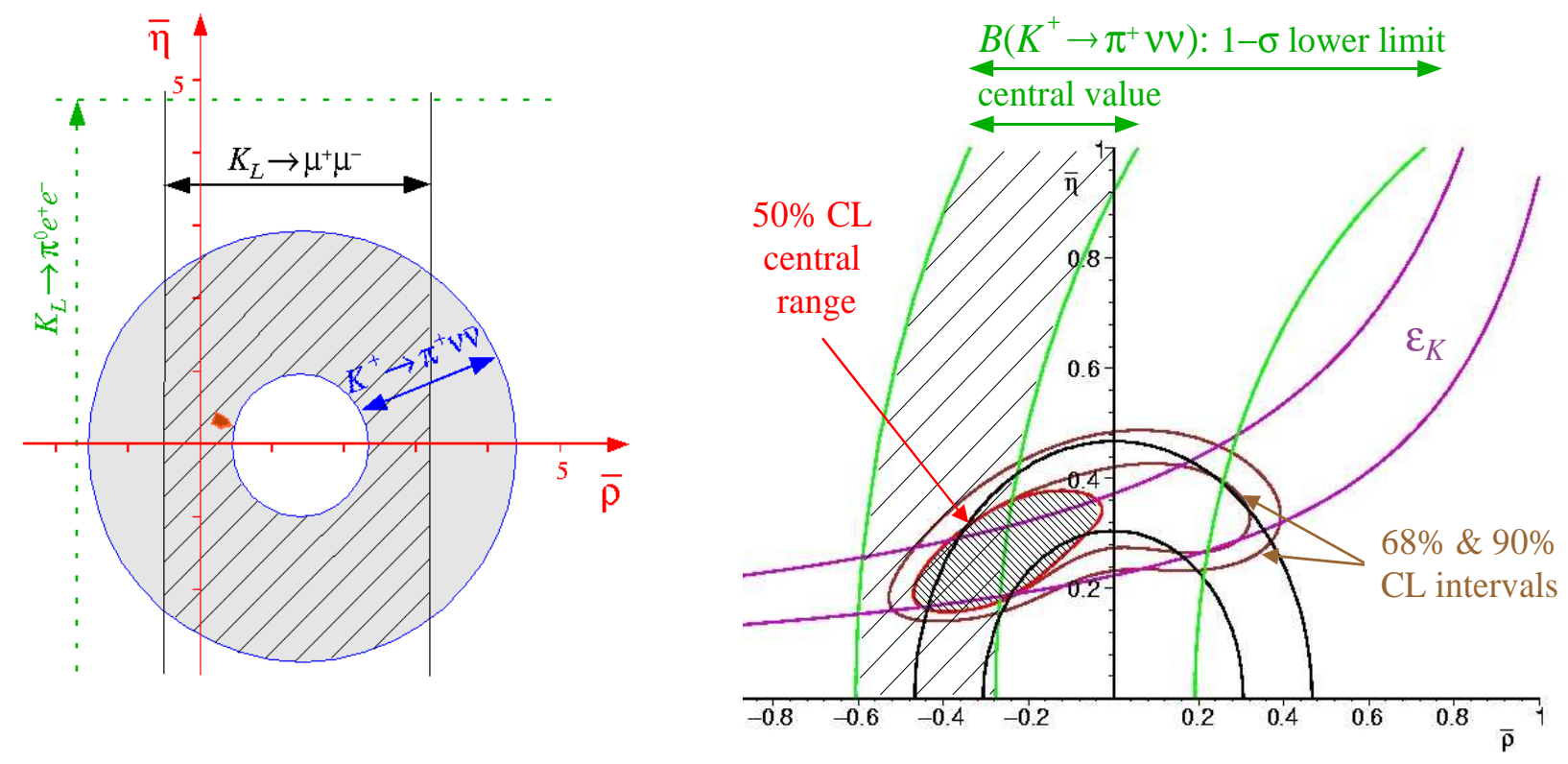

Figure 4: Left: present constraints in the $\bar{\rho}-\bar{\eta}$ plane from rare $K$ decays only (the small dark region close to the origin denotes the combined result of $B$-physics observables). Right: $\bar{\rho}-\bar{\eta}$ constraints excluding observables sensitive to $B_{d}-\bar{B}_{d}$ mixing [17].

good accuracy by the two-photon discontinuity and is calculable with high precision in terms of the $K_{L} \rightarrow \gamma \gamma$ rate. On the other hand, the dispersive contribution of the two-photon amplitude is a source of considerable theoretical uncertainties.

In the $K_{L} \rightarrow e^{+} e^{-}$mode the dispersive integral is dominated by a large infrared logarithm $\left[\sim \ln \left(m_{K}^{2} / m_{e}^{2}\right)\right]$, the coupling of which can be determined in a model-independent way from $\Gamma\left(K_{L} \rightarrow \gamma \gamma\right)$. As a result, $\Gamma\left(K_{L} \rightarrow e^{+} e^{-}\right)$can be estimated with good accuracy but is almost insensitive to short-distance dynamics 33 .

The $K_{L} \rightarrow \mu^{+} \mu^{-}$mode is certainly more interesting from the short-distance point of view. Here the two-photon long-distance amplitude is not enhanced by large logs and is almost comparable in size with the short-distance one, sensitive to $\Re \lambda_{t}[8]$. Actually short- and longdistance dispersive parts cancel each other to a good extent, since the total $K_{L} \rightarrow \mu^{+} \mu^{-}$ rate (measured with high precision by BNL-E871 [34]) is almost saturated by the absorptive two-photon contribution.

The accuracy on which we can bound the two-photon dispersive integral determines the accuracy of possible bounds on $\Re \lambda_{t}$. A partial control of the $K_{L} \rightarrow \gamma^{*} \gamma^{*}$ form factor, which rules the dispersive integral, can be obtained by means of $K_{L} \rightarrow \gamma \ell^{+} \ell^{-}$and $K_{L} \rightarrow e^{+} e^{-} \mu^{+} \mu^{-}$ spectra; additional constraints can also be obtained from model-dependent hadronic ansatze and/or perturbative QCD [35, 36]. Combining these inputs, significant upper bounds on $\Re \lambda_{t}$ (or lower bounds on $\bar{\rho}$ ) have recently been obtained [34, 37]. The reliability of these bounds has still to be fully investigated, but some progress can be expected in the near future. In particular, the extrapolation of the form factor in the high-energy region, which so far requires model-dependent assumptions, could possibly be limited by means of lattice calculations. 


\section{Beyond the MFV hypothesis}

To conclude this discussion about kaon physics, we summarize in Fig. 4 (left) the present impact of rare $K$ decays in constraining the $\bar{\rho}-\bar{\eta}$ plane. As can be noted, the bounds from these modes are substantially less precise than those from $B$-physics. As a result, we are still far from precision tests of the main MFV prediction: the universality of non-standard effects in $b \rightarrow d, b \rightarrow s$ and $s \rightarrow d$ FCNC transitions [6]. On the other hand, the comparison is already quite non-trivial concerning non-MFV scenarios. In particular, present rare- $K$-decay constrains put severe bounds on realistic scenarios with large new sources of flavour mixing in $s \rightarrow d$ transitions (see e.g. Ref. [38]).

Interestingly enough, non-MFV models with $\mathcal{O}(1)$ effects in $s \rightarrow d, b \rightarrow s$, and even $b \rightarrow d$ FCNC transitions are still far from being excluded. As an example, in Fig. 4 (right) we show the result of a fit allowing arbitrary new-physics contributions to $B_{d}-\bar{B}_{d}$ mixing. As can be noted, all remaining constraints are in good agreement; however, the large central value of $\mathcal{B}\left(K^{+} \rightarrow \pi^{+} \nu \bar{\nu}\right)$ tends to flavour a CKM structure rather different from the standard case [17. This indication is not statistically significant yet, but it provides a good illustration of the main points of this discussion: there is still a lot to learn about FCNC transitions and the measurements of $K \rightarrow \pi \nu \bar{\nu}$ rates provide a unique opportunity in this respect.

\section{Acknowledgments}

It is a pleasure to thank Giancarlo D'Ambrosio, Gian Giudice, and Alesandro Strumia for useful discussions and an enjoyable collaboration. I am also grateful to the organizers of TH2002 for the invitation to this interesting and unique conference. This work is partially supported by IHP-RTN, EC contract No. HPRN-CT-2002-00311 (EURIDICE).

\section{References}

[1] N. Cabibbo, Phys. Rev. Lett. 10, 531 (1963); M. Kobayashi and T. Maskawa, Prog. Theor. Phys. 49, 652 (1973).

[2] L. Wolfenstein, Phys. Rev. Lett. 51, 1945 (1983); A. J. Buras, M. E. Lautenbacher and G. Ostermaier, Phys. Rev. D 50, 3433 (1994).

[3] A. Stocchi, plenary talk at ICHEP 2002 (Amsterdam, Jul 2002), hep-ph/0211245.

[4] Y. Nir, plenary talk at ICHEP 2002 (Amsterdam, Jul 2002), hep-ph/0208080 G. Isidori, Int. J. Mod. Phys. A 17, 3078 (2002) hep-ph/0110255.

[5] R. S. Chivukula and H. Georgi, Phys. Lett. B 188, 99 (1987).

[6] G. D'Ambrosio, G. F. Giudice, G. Isidori and A. Strumia, Nucl. Phys. B 645, 155 (2002) hep-ph/0207036.

[7] A. Ali and D. London, Eur. Phys. J. C 9, 687 (1999) hep-ph/9903535; A. J. Buras et al., Phys. Lett. B 500, 161 (2001) hep-ph/0007085; A. Bartl et al., Phys. Rev. D 64, 076009 (2001) hep-ph/0103324; A. J. Buras and R. Fleischer, Phys. Rev. D 64, 115010 (2001) hep-ph/0104238; S. Laplace, Z. Ligeti, Y. Nir and G. Perez, Phys. Rev. D 65, 094040 (2002) hep-ph/0202010; C. Bobeth, T. Ewerth, F. Kruger and J. Urban, Phys. Rev. D 66, 074021 (2002) hep-ph/0204225.

[8] G. Buchalla and A. J. Buras, Nucl. Phys. B 398, 285 (1993); 400, 225 (1993); 412, 106 (1994) hep-ph/9308272 . 548, 309 (1999) hep-ph/9901288. 
[9] M. Misiak and J. Urban, Phys. Lett. B 451, 161 (1999) hep-ph/9901278.

[10] G. Buchalla and A. J. Buras, Phys. Rev. D 57, 216 (1998) hep-ph/9707243.

[11] A. F. Falk, A. Lewandowski and A. A. Petrov, Phys. Lett. B 505, 107 (2001) hep-ph/0012099.

[12] M. Lu and M. Wise, Phys. Lett. B 324, 461 (1994) hep-ph/9401204.

[13] L. Littenberg, Phys. Rev. D 39, 3322 (1989).

[14] G. Buchalla and A. J. Buras, Phys. Rev. D 54, 6782 (1996) hep-ph/9607447.

[15] G. Buchalla and G. Isidori, Phys. Lett. B 440, 170 (1998) hep-ph/9806501; D. Rein and L.M. Sehgal, Phys. Rev. D 39, 3325 (1989).

[16] W.J. Marciano and Z. Parsa, Phys. Rev. D 53, R1 (1996).

[17] G. D'Ambrosio and G. Isidori, Phys. Lett. B 530, 108 (2002) hep-ph/0112135.

[18] S. Kettell, L. Landsberg and H. Nguyen, hep-ph/0212321

[19] S. Adler et al. [E787 Collab.], Phys. Rev. Lett. 88, 041803 (2002) hep-ex/0111091; 84, 3768 (2000) hep-ex/0002015; 79, 2204 (1997) hep-ex/9708031;

[20] A. R. Barker and S. H. Kettell, Annu. Rev. Nucl. Part. Sci. 50, 249 (2000) hep-ex/0009024; A. Belyaev et al. [ECFA-CERN Kaon Physics Working Group], hep-ph/0107046 L. Littenberg, hep-ex/0212005

[21] J. Adams et al. [KTeV Collab.], Phys. Lett. B 447, 240 (1999) hep-ex/9806007.

[22] Y. Grossman and Y. Nir, Phys. Lett. B 398, 163 (1997) hep-ph/9701313.

[23] G. D'Ambrosio and G. Isidori, Int. J. Mod. Phys. A 13, 1 (1998) hep-ph/9611284.

[24] F. J. Gilman and M. B. Wise, Phys. Rev. D 21, 3150 (1980).

[25] G. Ecker, A. Pich and E. de Rafael, Nucl. Phys. B 291, 692 (1987).

[26] R. Appel et al. [E865 Collab.], Phys. Rev. Lett. 83, 4482 (1999) hep-ex/9907045.

[27] G. D’Ambrosio, G. Ecker, G. Isidori and J. Portolés, JHEP 08, 004 (1998).

[28] A. Lai et al. [NA48 Collab.], Phys. Lett. B 514, 253 (2001).

[29] A. J. Buras, M. E. Lautenbacher, M. Misiak and M. Munz, Nucl. Phys. B 423, 349 (1994) hep-ph/9402347.

[30] A. Alavi-Harati et al. [KTeV Collab.], Phys. Rev. Lett. 86, 397 (2001) hep-ex/0009030.

[31] L. M. Sehgal, Phys. Rev. D 38, 808 (1988); G. Ecker, A. Pich and E. de Rafael, Phys. Lett. B 237, 481 (1990); L. Cappiello, G. D'Ambrosio and M. Miragliuolo, Phys. Lett. B 298, 423 (1993); P. Heiliger and L.M. Sehgal, Phys. Rev. D 47, 4920 (1993); A G. Cohen, G. Ecker and A. Pich, Phys. Lett. B 304, 347 (1993); J.F. Donoghue and F. Gabbiani, Phys. Rev. D 51, 2187 (1995) hep-ph/9408390; G. D'Ambrosio and J. Portolés, Nucl. Phys. B 492, 417 (1997) hep-ph/9610244. F. Gabbiani and G. Valencia, Phys. Rev. D 66, 074006 (2002) hep-ph/0207189.

[32] A. Lai et al. [NA48 Collab.], Phys. Lett. B 536, 229 (2002) hep-ex/0205010.

[33] G. Valencia, Nucl. Phys. B 517, 339 (1998) hep-ph/9711377; G. Dumm and A. Pich, Phys. Rev. Lett. 80, 4633 (1998) [hep-ph/980129].

[34] D. Ambrose et al. [E871 Collab.], Phys. Rev. Lett. 84, 1389 (2000).

[35] L. Bergstrom, E. Masso and P. Singer, Phys. Lett. B 249, 141 (1990).

[36] G. D'Ambrosio, G. Isidori and J. Portolés, Phys. Lett. B 423, 385 (1998) hep-ph/9708326.

[37] A. Alavi-Harati et al. [KTeV Collab.], Phys. Rev. Lett. 87, 071801 (2001).

[38] A. J. Buras et al., Nucl. Phys. B 566, 3 (2000) hep-ph/9908371. 